\title{
Chapter 15 \\ DMP1 Binds Specifically to Type I \\ Collagen and Regulates Mineral Nucleation and Growth
}

\author{
Anne George, Elizabeth Guirado, and Yinghua Chen
}

\begin{abstract}
Extracellular matrix of bone and dentin is highly complex and involves a dynamic process of deposition and removal. Cells are the main architect that build this designer matrix that is highly specialized to calcified tissues. Osteoblasts or odontoblasts secrete both collagen and noncollagenous proteins in a temporal and spatial manner. Type I collagen self-assembles and forms a fabric-like template onto which noncollagenous proteins and mineral bind in a well-regulated manner. Dentin matrix protein 1 (DMP1) is one such noncollagenous protein that contains several acidic groups that can bind calcium ions which in turn binds phosphate and initiates the calcification process. In this study, we demonstrate that DMP1 is localized at specific sites on the self-assembled collagen matrix of dentin. In vitro nucleation studies on demineralized and deproteinized dentin slice adsorbed with DMP1 show bundles of well-ordered needle-shaped nanohydroxyapatite deposited on the dentin matrix. The nucleated mineral structures had uniform length and width and their long axis was oriented parallel to the collagen fibril axis. Overall, the physiologically selfassembled collagen and DMP1 mediated ordered deposition of nanocrystalline HAP.
\end{abstract}

Keywords Dentin matrix protein $1 \cdot$ Collagen $\cdot$ Hydroxyapatite $\cdot$ Mineralization $\cdot$ Extracellular matrix

\subsection{Introduction}

Biological composites such as bone, dentin, and cementum consist of a crystalline inorganic phase mainly carbonated hydroxyapatite embedded within a biopolymeric organic matrix (Veis 1993; Veis and Dorvee 2013). The cells that produce these mineralized matrices exert a regulatory and exquisite control over the minerals they deposit, creating materials of varied shapes, sizes, and high tensile strength.

\footnotetext{
A. George $(\bowtie) \cdot$ E. Guirado · Y. Chen

Brodie Tooth Development Genetics \& Regenerative Medicine Research Laboratory, Department of Oral Biology, University of Illinois at Chicago, Chicago, IL, USA

e-mail: anneg@uic.edu; eguira@uic.edu; yinghua@uic.edu
} 
The stereospecific interaction of macromolecules with biominerals represents a unique phenomenon in nature (Addadi et al. 2001; Grzesiak et al. 2017).

Mineralized tissue formation results from the coordinated activity of highly differentiated cells. During the differentiation process, these cells secrete an extracellular matrix which performs various functions (Grzesiak et al. 2017; Hirata et al. 2005; Kang et al. 2015). A distinct feature of the ECM in calcified tissues is that it contains both crystal growth promoters and inhibitors (Goldberg and Smith 2004). Components in the ECM are involved in directing the deposition of specific calcium phosphate polymorphs during the formation of the calcified matrix.

The deposition of extracellular matrix and subsequent mineralization are a temporal and spatial event. They are assembled from a collagenous fibrillar network containing small amounts of tissue-specific regulatory proteins and other widely circulatory proteins. Phosphoproteins are one of the major component groups among the noncollagenous proteins found in all calcified tissues (Ravindran and George 2014). They have been postulated to play an important role in the initiation of calcification and possibly in the regulation of crystal growth. These regulatory proteins are responsible for controlled crystal growth within the collagenous matrix.

Dentin matrix protein (DMP1) is an example of a regulatory protein localized in the ECM of bone and dentin (George et al. 1993, 1994, 1995; He and George 2004). Understanding the players and the mechanism by which the extracellular matrix calcifies is important to understand the function of mineralized biological tissues. In the current study, we demonstrate that DMP1 is localized to specific sites on the dense physiologically arranged collagen fibrils of demineralized dentin matrix. Using a demineralized and deproteinized dentin slice, we demonstrate that adsorbed recombinant DMP1 can promote the deposition of organized nanocrystalline hydroxyapatite ribbons. Modeling of DMP1 shows that the protein could have a flexible carboxyl terminal domain, which might help in binding collagen and $\mathrm{Ca}^{2+}$ in the ECM.

\subsection{Methods}

\subsubsection{Expression of Recombinant DMP1 Protein}

Full-length rat recombinant DMP1 was produced as previously published (BedranRusso et al. 2013; Srinivasan et al. 1999). BSA (bovine serum albumin) was used as a negative control.

\subsubsection{Preparation of Demineralized Dentin Wafers for Immunogold Labeling}

Third molars without caries were selected and kept frozen following approval of the Institutional Review Board at the University of Illinois at Chicago (protocol 20090198). Coronal dentin cross-sections $1.5 \mathrm{~mm}$ thick were cut from each tooth and 
further sectioned to produce 250 - $\mu$ m-thick slices using a hydrated diamond blade saw (IsoMet 1000, Buehler, Lake Bluff, IL, USA). These slices represent the samples hereinafter referred to as dentin wafers.

Wafers measuring $1.5 \times 3 \times 0.250 \mathrm{~mm}$ thick were placed together in $14 \%$ EDTA at $4{ }^{\circ} \mathrm{C}$ for 10 days. Demineralization was verified by X-ray microradiography (MX-20 Faxitron, LLC, Lincolnshire, IL). These dentin wafers were dehydrated in a series of ethanol from $30 \%$ to $100 \%$, trimmed, and embedded in epoxy resin, and ultrathin sections $70 \mathrm{~nm}$ were placed on 300 mesh formvar-coated nickel grids.

Grid-mounted tissue sections were processed for colloidal gold immunohistochemistry by incubating the sections with rabbit primary antibody against DMP1 (1:250) as published before. After which the sections were incubated with anti-rabbit IgG colloidal gold particles (10 nm gold particles) and washed. For controls, the sections were incubated with $20 \mathrm{~nm}$ gold-conjugated anti-rabbit IgG. All the sections were imaged either unstained or stained with uranyl acetate and imaged using JEOL JEM 1220 Electron Microscope and digital images obtained using Erlangshen ESW 1000w 785 camera.

\subsubsection{Preparation of Demineralized and Deproteinized Dentin Wafers for Nucleation Experiments}

Demineralized dentin wafers were subjected to treatment with $1 \mathrm{M} \mathrm{NaCl}$ for $1 \mathrm{~h}$ at room temperature to disrupt loosely bound noncollagenous proteins. Samples were then incubated in $0.25 \%$ trypsin-EDTA twice for $4 \mathrm{~h}$ at $37{ }^{\circ} \mathrm{C}$ to remove strongly bound, endogenous noncollagenous proteins. These wafers were cryosectioned to $5 \mu \mathrm{m}$, stained with Stains-All ${ }^{\circledR}$ (Sigma-Aldrich), and imaged to confirm the completeness of removal of noncollagenous proteins (Padovano et al. 2015).

In vitro nucleation was performed as reported earlier for 14 days (He et al. 2003). At the end of the time point, samples were washed and dehydrated by passing through a series of graded ethanol solutions, 30\%, 50\%, 90\%, and $100 \%$ for $10 \mathrm{~min}$ each. The samples were finally dehydrated by immersing them in a solution of hexamethyldisilazane embedded in epoxy resin, and ultrathin sections of $70 \mathrm{~nm}$ were placed on copper TEM grid and examined in a JEOL JEM 1220 TEM (JEOL Ltd., Tokyo, JAPAN) Gatan accelerating voltage of $60 \mathrm{kV}$. Images were recorded using a CCD camera (Gatan Inc., Pleasanton, CA).

\subsubsection{Modeling of DMP1}

The I-TASSER (Iterative Threading ASSEmbly Refinement) server was used for DMP1 3D structure prediction (Roy et al. 2010; Yang et al. 2015; Yang and Zhang 2015a, b; Zhang 2009). The polypeptide strand was built by using human DMP1 sequence data from NCBI protein database (NP_004398.1). The program uses ab 
initio modeling as well as the LOMETS multiple threading program to retrieve proteins with similar folds from the PDB library. Replica-exchange Monte Carlo simulations generated a pool of protein structures from which low free-energy states were selected. Iterative template fragment assembly simulations identified the top five near-native models. C scores between -5 and 2 are assigned to these models, with more positive scores indicating greater confidence.

\subsection{Results}

\subsubsection{DMP1 Binds to Densely Packed Collagen Fibrils of the Dentin Matrix}

Immunogold labeling experiment showed that gold particles were distributed specifically over the dense collagen fibril of the demineralized dentin matrix (Fig. 15.1a). Demineralization of dentin led to the dissolution of the mineral only and did not alter the antigenic properties of DMP1 in the matrix. Staining the dentin slice with uranyl acetate and lead citrate showed that DMP1 was localized on the collagen matrix. Image presented in Fig. 15.1b shows that the labeling was mostly localized at the edge of the $67 \mathrm{~nm}$ periodic band of type I collagen. This region would correspond to the gap region of the self-assembled collagen matrix.
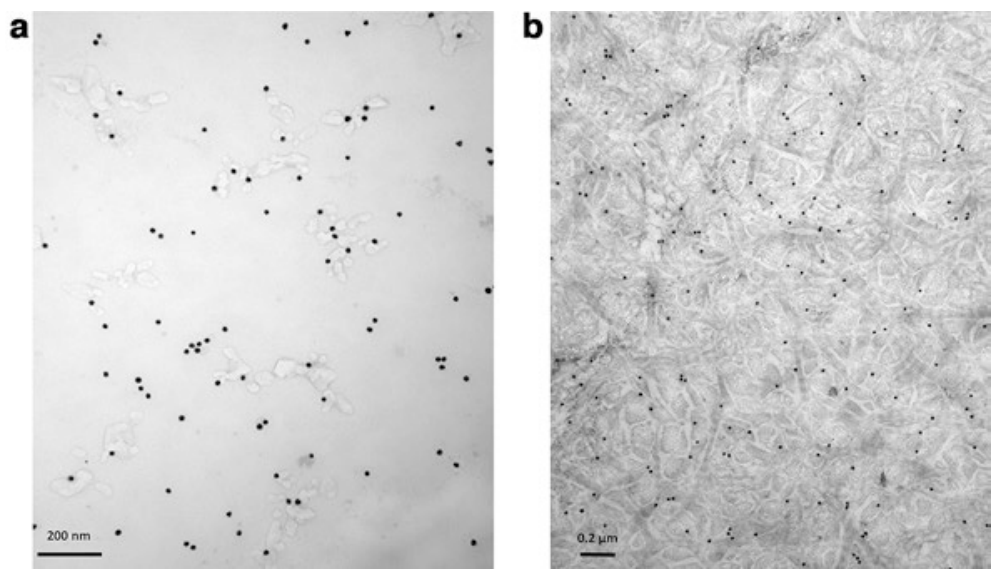

Fig. 15.1 Localization of DMP1 on the dentin wafer. (a) Unstained transmission electron micrograph of the demineralized dentin slice on which immunogold labeling with anti-DMP1 was performed prior to TEM. Scale bar represents $200 \mathrm{~nm}$. (b) Same experiment wherein the dentin slice was stained with uranyl acetate and lead citrate. Scale bar represents $0.2 \mu \mathrm{m}$ 

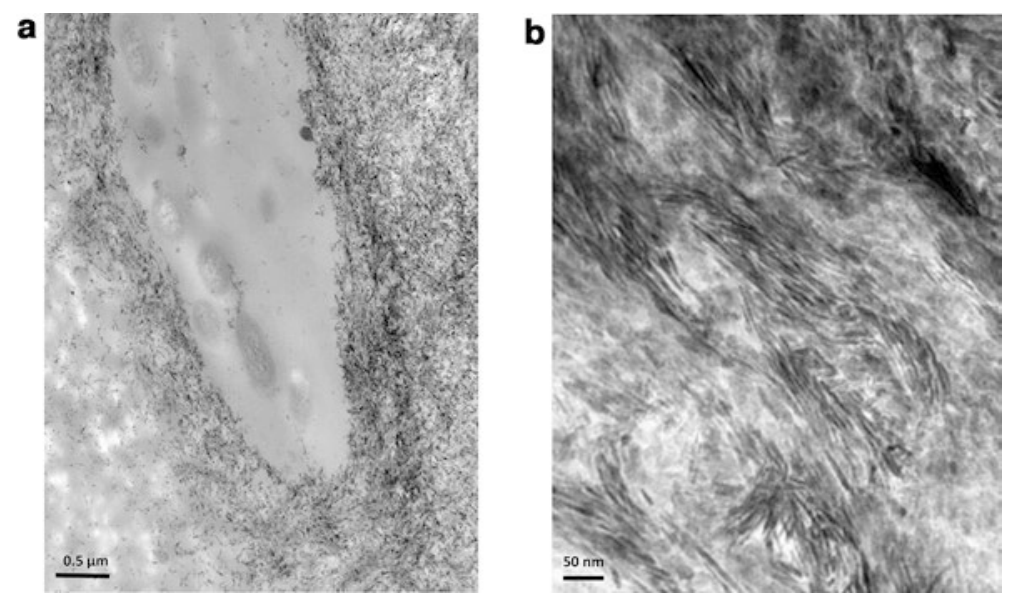

Fig. 15.2 Structural morphology of DMP1 mediated mineral deposition: (a) transmission electron microscopy (TEM) image of DMP1 mediated HA nanocrystal on demineralized and deproteinized dentin slice. Scale bar represents $0.5 \mu \mathrm{m}$. (b) Higher magnification image showing bands of closely aligned mineral fibers aligned parallel to the collagen fibrils. Scale bar represents $50 \mathrm{~nm}$

\subsubsection{Structural Characterization of the Mineral Deposited on the Collagen Matrix of Dentin}

In vitro nucleation studies showed that demineralized and deproteinized dentin slices adsorbed with DMP1 initiated calcium phosphate deposition. At the end of 14 days, TEM analysis showed the presence of nanostructured mineral structures which were aligned to the dentin collagen fibrils (Fig. 15.2). At low magnification (Fig. 15.2a), it was apparent that the dentin slice was coated with electron-dense calcium phosphate mineral, particularly concentrated around the dentinal tubule. The high magnification image (Fig. 15.2b) shows that the dark electron-dense deposits are bundles of mineral lamellae which are about 3-5 $\mathrm{nm}$ apart. The needlelike mineral lamellae were all oriented with their long axis nearly parallel to the collagen fibrils. Selected area electron diffraction analysis (SAED) of the acicular deposits (Fig. 15.3a) showed the presence of nanocrystalline hydroxyapatite as they displayed the 002 reflection pattern (Fig. 15.3b).

\subsubsection{Computation and Ab Initio Models of DMP1}

Computer modeling was used to gain insight into the molecular shape of DMP1. Two predicted models are shown in Fig. 15.4a, b. Of these, (b) represents the highest scoring model $\mathrm{C}$-score of $(-1.59)$, while, model a had a C-score of $(-2.54)$. 

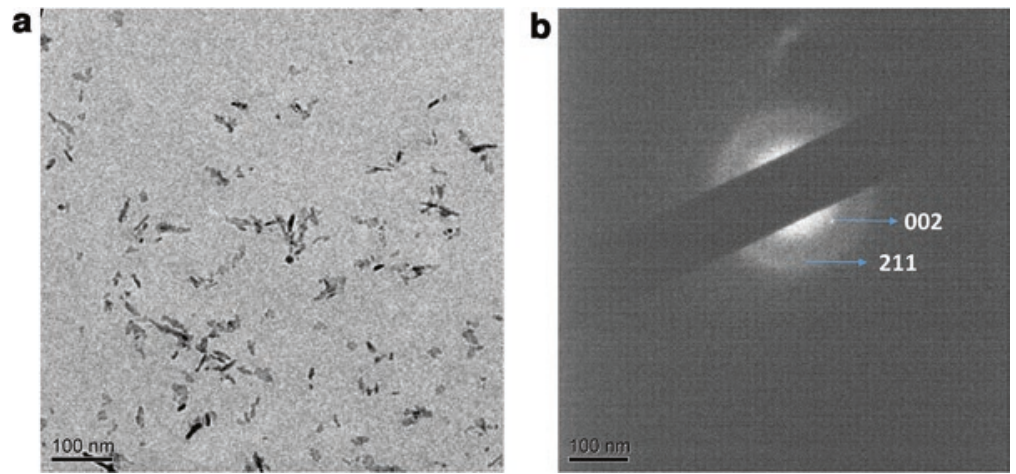

Fig. 15.3 Characterization of the Ca-P deposits: (a) TEM image shows nanocrystalline Ca-P deposits with acicular morphology. (b) Selected area electron diffraction pattern indexed to nanocrystalline HAP. Note that the 002 reflections are in the process of forming arcs indicating preferred orientation of the c-axis of the HAP parallel to that of the collagen fibrils

a

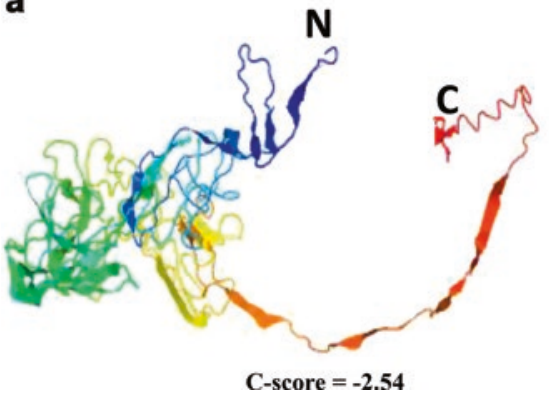

b

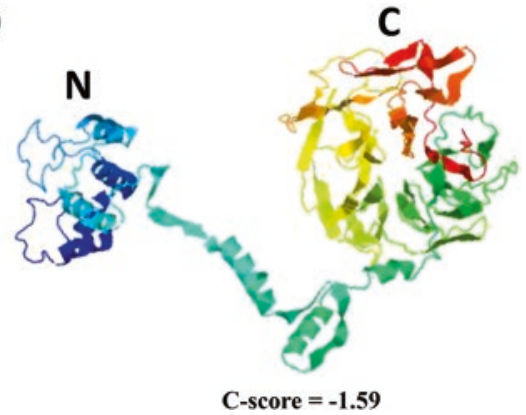

Fig. 15.4 Energy-minimized models of full-length DMP1: the I-TASSER (Iterative Threading ASSEmbly Refinement) server was used for DMP1 3D structure prediction (NP_004398.1). The program uses ab initio modeling as well as the LOMETS multiple threading program to retrieve proteins with similar folds from the PDB library

Model a shows a "globular" N-terminus with an "extended arm" at the C-terminus. However, Model $b$ with a lower free-energy shows that the $\mathrm{C}$-terminus had the propensity to fold into beta sheets, while the N-terminus remained globular.

\subsection{Discussion}

The constituents of the extracellular matrix of bone and dentin are responsible for modulating nucleation of apatite nanoparticles and their growth into micrometersized crystals. The organic matrix mainly consists of type I collagen which forms the template that directs ordered deposition of mineral crystals. The rest of the 
components of the organic matrix consist of noncollagenous proteins, lipids, and proteoglycans. Each of these classes of macromolecules play decisive roles in the controlled growth of HAP on the collagen matrix.

In this study, we used the demineralized and deproteinized dentin slice as a template to demonstrate the role of extracellular dentin matrix protein 1, a member of the noncollagenous class of proteins in calcified tissues. The processed dentin slice contains fibrillar cross-linked collagen arranged in a three-dimensional supramolecular architecture.

We first showed the spatial localization of DMP1 on the demineralized collagen matrix. Demineralization was able to expose the epitope site for the antibody to bind to DMP1. Gold particles were specifically localized on the collagen fibril. As DMP1 is a calcium-binding protein, there is a good possibility that some might be removed from the dentin matrix during the process of demineralization. Specific localization of DMP1 on the collagen template suggests that initiation of mineral deposition would be site specific.

In vitro nucleation experiments conducted on the dentin wafer adsorbed with DMP1 show abundant Ca-P deposits around the dentinal tubule. Higher magnification of the deposits shows that nucleation is not a random event, but the process is initiated in spatially distinct nucleation sites. Closer inspection shows that the nanocrystals are deposited as bundles of mineral structures in a highly organized manner with their c-axis oriented nearly parallel to the collagen fibrils. The nanocrystalline deposits had nearly similar length and widths suggesting that the self-assembled collagen fibrils could dictate the morphology and growth of the mineral deposits. The mineral structures were characterized as nanocrystalline HAP. McNally et al. also reported the presence of similar rigid hydroxyapatite struts encased in the collagen matrix of the human femoral bone (McNally et al. 2012). In a new model for the ultrastructure of bone based on TEM analysis, Schwarcz reports the presence of stacks of mineral lamellae packed around the fibrils and that the lamellae are spaced less than $1 \mathrm{~nm}$ apart (Schwarcz 2015). Similar ultrastructure of the mineral phase was observed in this study.

With the use of molecular modeling, we have demonstrated that DMP1 contains a flexible loop between the $\mathrm{C}$ - and $\mathrm{N}$-termini where the proteolytic cleavage site resides. The stringent model (Fig. 15.4b) showed that C-DMP1 had the propensity to form beta sheets. This could be envisaged as DMP1 in the presence of calcium adopts a beta sheet structure (He et al. 2003). We have shown that the C-terminal portion of DMP1 is highly acidic due to abundant glutamic acid residues along with serines which could be phosphorylated, thereby increasing the negative charge (He et al. 2005). We have also demonstrated that intermolecular assembly of the peptides at the $\mathrm{C}$-terminus in the presence of calcium ions was important to form a stereospecific template for hydroxyapatite nucleation. It is possible that the nanocrystalline hydroxyapatite fibrillar deposits observed in this study could be dependent on the beta sheet conformation of C-DMP1 and the confined gap regions present in the three-dimensional supramolecular architecture of self-assembled type I collagen. 
Overall, this study demonstrates that biomineralization is a complex cellmediated process and the DMP1 has an intimate relationship with the collagen matrix. Such an interaction dictates the site-specific nucleation of Ca-P nanocrystalline hydroxyapatite. Subsequently, other proteins in the ECM might be responsible for crystal fusion and controlled growth of the initially nucleated crystals.

Acknowledgments This work was supported by National Institutes of Health Grant DE 11657 and the Brodie Endowment Fund (AG). "This work made use of instruments in the Electron Microscopy Service (Research Resources Center, UIC."

\section{References}

Addadi L, Weiner S, Geva M (2001) On how proteins interact with crystals and their effect on crystal formation. Z Kardiol 90(3):92-98

Bedran-Russo AK, Ravindran S, George (2013) A imaging analysis of early DMP1 mediated dentine remineralization. Arch Oral Biol 58:254-260

George A, Sabsay B, Simonian PA, Veis A (1993) Characterization of a novel dentin matrix acidic phosphoprotein. Implications for induction of biomineralization. J Biol Chem 268:12624-12630

George A, Gui J, Jenkins NA, Gilbert DJ, Copeland NG, Veis A (1994) In situ localization and chromosomal mapping of the AG1 (Dmp1) gene. J Histochem Cytochem 42:1527-1531

George A, Silberstein R, Veis A (1995) In situ hybridization shows Dmp1 (AG1) to be a developmentally regulated dentin-specific protein produced by mature odontoblasts. Connect Tissue Res 33:67-72

Goldberg M, Smith AJ (2004) Cells and extracellular matrices of dentin and pulp: a biological basis for repair and tissue engineering. Crit Rev Oral Biol Med 15:13-27

Grzesiak J, Smieszek A, Marycz K (2017) Ultrastructural changes during osteogenic differentiation in mesenchymal stromal cells cultured in alginate hydrogel. Cell Biosci 7:2

He G, George A (2004) Dentin matrix protein 1 immobilized on type I collagen fibrils facilitates apatite deposition in vitro. J Biol Chem 279:11649-11656

He G, Dahl T, Veis A, George A (2003) Dentin matrix protein 1 initiates hydroxyapatite formation in vitro. Connect Tissue Res 44(1):240-245

He G, Gajjeraman S, Schultz D, Cookson D, Qin C, Butler WT, Hao J, George A (2005) Spatially and temporally controlled biomineralization is facilitated by interaction between selfassembled dentin matrix protein 1 and calcium phosphate nuclei in solution. Biochemistry 44:16140-11614

Hirata I, Nomura Y, Tabata H, Miake Y, Yanagisawa T, Okazaki M (2005) SEM observation of collagen fibrils secreted from the body surface of osteoblasts on a CO3apatite-collagen sponge. Dent Mater J 24:460-464

Kang H, Shih YR, Varghese S (2015) Biomineralized matrices dominate soluble cues to direct osteogenic differentiation of human mesenchymal stem cells through adenosine signaling. Biomacromolecules 16:1050-1061

McNally EA, Schwarcz HP, Botton GA, Arsenault AL (2012) A model for the ultrastructure of bone based on electron microscopy of ion-milled sections. PLoS One 7:e29258

Padovano JD, Ravindran S, Snee PT, Ramachandran A, Bedran-Russo AK, George A (2015) DMP1-derived peptides promote remineralization of human dentin. J Dent Res 94:608-614

Ravindran S, George A (2014) Multifunctional ECM proteins in bone and teeth. Exp Cell Res 325:148-154

Roy A, Kucukural A, Zhang Y (2010) I-TASSER: a unified platform for automated protein structure and function prediction. Nat Protoc 5:725-738 
Schwarcz HP (2015) The ultrastructure of bone as revealed in electron microscopy of ion-milled sections. Semin Cell Dev Biol 46:44-50

Srinivasan R, Chen B, Gorski JP, George A (1999) Recombinant expression and characterization of dentin matrix protein 1 . Connect Tissue Res 40:251-258

Veis A (1993) Mineral-matrix interactions in bone and dentin. J Bone Miner Res 8(2):S493-S497

Veis A, Dorvee JR (2013) Biomineralization mechanisms: a new paradigm for crystal nucleation in organic matrices. Calcif Tissue Int 93:307-315

Yang J, Zhang Y (2015a) I-TASSER server: new development for protein structure and function predictions. Nucleic Acids Res 43:W174-W181

Yang J, Zhang Y (2015b) Protein structure and function prediction using I-TASSER. Curr Protoc Bioinformatics 52 5(8):1-15

Yang J, Yan R, Roy A, Xu D, Poisson J, Zhang Y (2015) The I-TASSER suite: protein structure and function prediction. Nat Methods 12:7-8

Zhang Y (2009) I-TASSER: fully automated protein structure prediction in CASP8. Proteins 77(9):100-113

Open Access This chapter is licensed under the terms of the Creative Commons Attribution 4.0 International License (http://creativecommons.org/licenses/by/4.0/), which permits use, sharing, adaptation, distribution and reproduction in any medium or format, as long as you give appropriate credit to the original author(s) and the source, provide a link to the Creative Commons license and indicate if changes were made.

The images or other third party material in this chapter are included in the chapter's Creative Commons license, unless indicated otherwise in a credit line to the material. If material is not included in the chapter's Creative Commons license and your intended use is not permitted by statutory regulation or exceeds the permitted use, you will need to obtain permission directly from the copyright holder. 